\title{
Low Mass Standard Model Higgs Boson Searches at the Tevatron
}

\author{
Andrew Mehta*t \\ University of Liverpool \\ E-mail: mehta@hep.ph.liv.ac.uk
}

A search for the standard model Higgs boson in the low Higgs mass region $\left(M_{H}<135 \mathrm{GeV}\right)$ is presented using data from the Tevatron experiments CDF and D0 at a centre of mass energy of $1.96 \mathrm{TeV}$. The analyses presented here use an integrated luminosity of $0.9-2.7 \mathrm{fb}^{-1}$ of data. Several channels with different Higgs production mechanisms and decays are investigated. The channels that have the highest sensitivity are associated production of the Higgs with either a $W$ or $Z$ boson, where the Higgs decays to a $b \bar{b}$ quark pair and the vector boson decays leptonically. No signal is observed and limits on the Higgs production cross section of between 4-7 times that predicted by the standard model have been placed.

2008 Physics at LHC

September 29 - 4 October 2008

Split, Croatia

\footnotetext{
* Speaker.

${ }^{\dagger}$ representing the CDF and D0 collaborations.
} 


\section{Introduction}

The mechanism of electroweak symmetry breaking, as described in standard model (SM), requires the existence of a scalar, neutral particle, the Higgs boson. Direct searches at the LEP collider showed that the mass should be $M_{H}>114.4 \mathrm{GeV}$ [1]. The Higgs can also be detected indirectly via loop corrections to electroweak processes; a compilation of measurements at various experiments, including LEP and the Tevatron, yield a constraint of $M_{H}<154 \mathrm{GeV}$ at $95 \%$ confidence level [2].

At the present time the Tevatron, which collides, protons and anti-protons at a centre of mass energy of $1.96 \mathrm{TeV}$, is the only place where Higgs bosons can be produced. The Tevatron is running well with data corresponding to an integrated luminosity of $\sim 4 \mathrm{fb}^{-1}$, recorded by each of the two experiments CDF and D0. This paper reviews those analyses that are sensitive to the production of a low mass Higgs $\left(M_{H}<135 \mathrm{GeV}\right)$. The analyses use $0.9-2.7 \mathrm{fb}^{-1}$ of data per experiment. SM Higgs searches at higher masses are reviewed in [3].

Large numbers of Higgs bosons will be produced at the Tevatron if its mass is low. Unfortunately the dominant decay of $H \rightarrow b \bar{b}$ is swamped by background - heavy flavour production of jets is $\sim 9$ orders of magnitude larger. The production of a Higgs boson in association with a $W$ or $Z$ boson, however, has lower backgrounds. Events where the vector vector boson decays leptonically and the Higgs decays to $b \bar{b}$ have the most sensitivity for discovery of a low mass SM Higgs. Several other channels, which have smaller sensitivity have also been investigated by the Tevatron experiments. It should also be noted that the channel $H \rightarrow W^{+} W^{-}$, which is discussed in [3], also contributes to the sensitivity in the low mass region. Further details on the analyses presented here and the latest results can be obtained from [4].

\section{Lepton and missing transverse momentum reconstruction}

Electrons and muons from $W$ and $Z$ decays are reconstructed with a transverse momentum typically $p_{T} \gtrsim 15 \mathrm{GeV}$. Considerable effort has been made to extend the acceptance of leptons into the higher $|\eta|$ regions, and most analyses now accept lepton with $|\eta| \lesssim 2.0$. The missing transverse energy $Z_{T}$ is used to tag neutrinos that do not make energy deposits in the detector. The $E_{T}$ is reconstructed from all hadronic energy deposits and the energy of all reconstructed leptons in the event. Typically a requirement of $E_{T} \gtrsim 20 \mathrm{GeV}$ is placed to tag a neutrino from $W$ decay. The signals from electrons, muons and $H_{T}$ are the main way events are triggered in the analyses described here.

\section{3. $b$ tagging}

One of the main ways of identifying the Higgs through it's dominant decay $H \rightarrow b \bar{b}$ is to identify one or both the final state $b$ jets. Since $b$ hadrons have a relatively long lifetime the decay products can be "tagged" by reconstructing a secondary vertex or identifying tracks displaced from the vertex. This is achieved by the precise spatial reconstruction of tracks in the silicon tracking detectors of the two experiments.

CDF uses several different approaches to tag $b$ jets. The standard method is to explicitly reconstruct a secondary vertex using two or more tracks. An alternative method is to work out the 

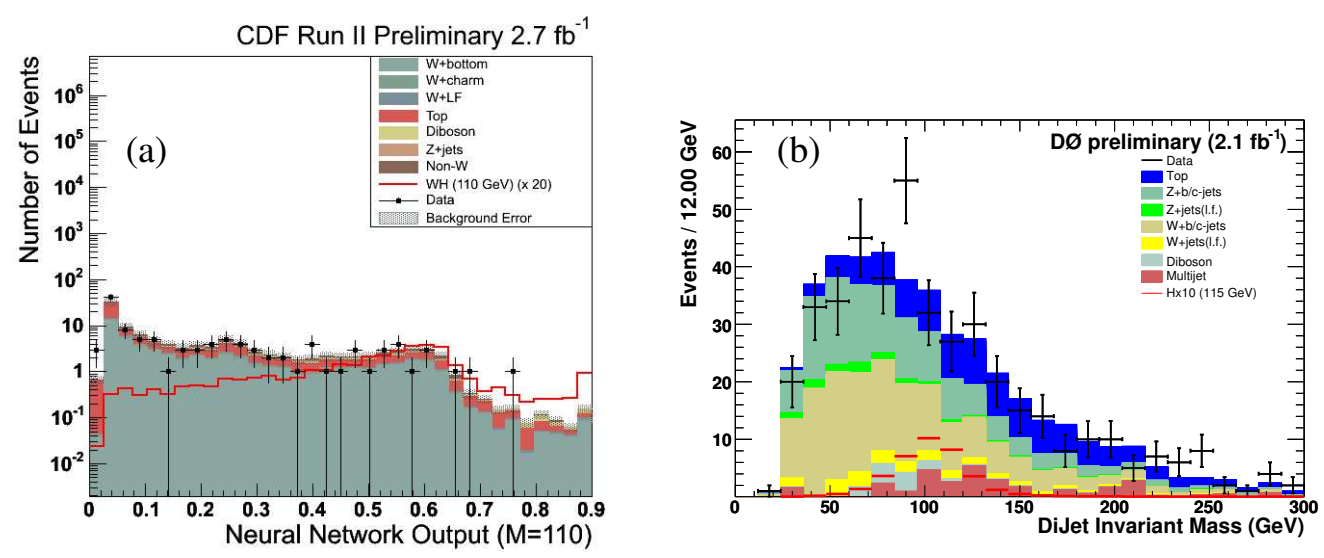

Figure 1: (a) The NN output from the CDF analysis of the $W H$ channel with two tags. (b) The dijet invariant mass for the $Z H \rightarrow v \bar{v} b \bar{b}$ channel. The distributions from the Higgs expectation have been scaled for visual clarity.

probability that all tracks in the jet originated at the primary vertex using the impact parameter of tracks. They also combine several track and jet observables in an artificial neural network (NN). D0 combines secondary vertex and track impact parameter variables, together with quantities designed to reconstruct the leptons from semileptonic $b$ decays in a NN. Using the $\mathrm{NN}$ a typical tagging efficiency of $50 \%$ is achieved, with a mistag rate of $0.5 \%$ for jets of $p_{T}=50 \mathrm{GeV}$.

\section{The $W H$ associated production channel, $W H \rightarrow l v b \bar{b}$}

The $W$ in this channel is tagged by requiring an electron or muon together with $E_{T}$. The Higgs is reconstructed by requiring exactly two jets in addition to the $W$. The events are divided into two samples with one or two tagged jets. CDF has analysed $2.7 \mathrm{fb}^{-1}$ of data; D0 has analysed $1.7 \mathrm{fb}^{-1}$.

Background, which mainly arises from $W+$ jets and $t \bar{t}$ production, is suppressed by combining several kinematic variables in a NN. Variable that are used include the $p_{T}$ of the jets, the interjet separation and the invariant mass of the jets. An example $\mathrm{NN}$ output is displayed in figure 1 (a). Other multi-variant techniques such as a matrix element combined with a boosted decision tree have also been tested. This channel is currently the most sensitive channel at low mass; CDF observes a limit of $5.0 \times$ SM Higgs cross section ${ }^{1}$ and D0 observes $9.3 \times$ SM Higgs cross section.

D0 have, in addition, a dedicated analysis of the $W H \rightarrow \tau v b \bar{b}$ channel. A hadronic $\tau$ is reconstructed, along with $H_{T}$ and two $b$ jets. The dijet invariant mass is used to extract the limit of $35 \times$ SM Higgs cross section for $0.9 \mathrm{fb}^{-1}$ of data.

\section{The $Z H$ associated production channel, $Z H \rightarrow v \bar{v} b \bar{b}$}

Events are reconstructed by requiring $\phi_{T}>50 \mathrm{GeV}$ in addition to one or two tagged jets. At most one other jet is allowed in addition to the two jets assumed to come from the Higgs. Further cuts, mainly based on the angles of the jets, are used to suppress background from multi-jet production. Both collaborations have analysed $2.1 \mathrm{fb}^{-1}$ of data.

\footnotetext{
${ }^{1}$ All limits presented in this paper are at $95 \%$ confidence level.
} 
The signal for this channel contains a large fraction of events from $W^{ \pm} H \rightarrow l^{ \pm} v b \bar{b}$, where the lepton has not been properly reconstructed. Allowing a third jet in this analysis improves acceptance for the decay $W H \rightarrow \tau \nu b \bar{b}$.

The dominant backgrounds in this channel arises from multi-jet production and $W / Z+$ jet events. It is suppressed using multi-variant techniques of NN (CDF) and a boosted decision tree (D0). The invariant mass of the dijet system for the D0 analysis is shown in figure 1 (b). CDF observes a limit of $7.9 \times$ SM Higgs cross section and D0 observes 7.5 $\times$ SM Higgs cross section.

\section{The $Z H$ associated production channel, $Z H \rightarrow l^{+} l^{-} b \bar{b}$}

Events in this channel are selected by requiring two electrons or muons in addition to one or two tagged jets. Since this channel is fully reconstructed with no expected $E_{T}$, CDF uses the over-constraints of the kinematics to improve the resolution of the dijet invariant mass. CDF has analysed $2.4 \mathrm{fb}^{-1}$ of data; D0 has analysed $2.3 \mathrm{fb}^{-1}$.

As in the other channels multi-variant techniques are employed to improve the sensitivity. CDF observes a limit of $11.6 \times$ SM Higgs cross section and D0 observes $11.0 \times$ SM Higgs cross section.

\section{Other channels}

Other channels have also been investigated, which although not as sensitive can add to the overall significance and, could yield a signal if the Higgs' couplings to other particles was not as predicted by the SM.

The channel $V H \rightarrow q \overline{q^{\prime}} b \bar{b}$, where $V$ is a $W$ or $Z$ boson, has a large cross section due to the higher hadronic branching fraction of the $W$ and $Z$, but also has larger background from multi-jet production. In the analysis by CDF four jets are required, two of which are tagged. The dominant multi-jet background is estimated using a data driven method. A limit of $37 \times$ SM Higgs cross section for $2.0 \mathrm{fb}^{-1}$ is obtained.

The channel $t \bar{t} H \rightarrow l v b \bar{b} q \bar{q} b \bar{b}$ is the most complicated final state of any SM Higgs search at the Tevatron. In the analysis by D0 one lepton, $E_{T}$ and $\geq 4$ jets are required. The events are separated into samples based on jet and tag multiplicity. A limit of $64 \times$ SM Higgs cross section for $2.1 \mathrm{fb}^{-1}$ is obtained.

CDF performs a search for the decay $H \rightarrow \tau^{+} \tau^{-}$in association with two jets. This is a simultaneous search of the $V H$, vector boson fusion and $g g \rightarrow H$ channels. One tau is reconstructed via its leptonic decay and the other by its hadronic decay. A limit of $31 \times$ SM Higgs cross section for $2.2 \mathrm{fb}^{-1}$ is obtained.

The branching fraction of $H \rightarrow \gamma \gamma$ is rather low, but the channel is relatively clean leading to a peak in the diphoton invariant mass. A search has been performed by D0 using a NN to separate photons from $\pi^{0} \mathrm{~s}$ and other hadrons. A limit of $31 \times$ SM Higgs cross section for $2.7 \mathrm{fb}^{-1}$ is obtained. 


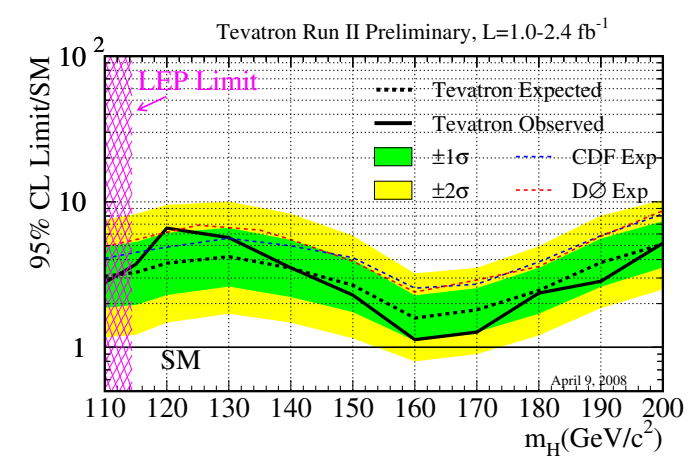

Figure 2: The combined CDF D0 cross section limit divided by the SM Higgs cross section shown as a function of mass. The results are from April 2008.

\section{Combined Results}

All the channels are combined to provide a cross section limit as a function of $M_{H}$. At $M_{H}=$ $115 \mathrm{GeV}$ the $\mathrm{CDF}^{2}$ only limit is now $3.2 \times \mathrm{SM}$ with about $2.4 \mathrm{fb}^{-1}$. Unfortunately, due the large number of channels at low mass, at the time of writing a CDF/D0 combination using the latest results is not yet available. The results from April 2008 are shown in 2. It is expected that the limit for $M_{H}=115 \mathrm{GeV}$ will fall to below $3 \times$ the SM when all the latest data are included in the combination.

\section{Summary and Outlook}

Higgs searches at the Tevatron are a challenge due to the small signal cross section and large backgrounds. The experiments have shown, however, that with a good understanding of the detector, using multi-variant techniques and combination of several channels, it should be possible to have sensitivity to a low mass SM Higgs with more data. By the end of data taking a total of 6-8 $\mathrm{fb}^{-1}$ of data should have been recorded by each experiment. If the experiments continue to improve their analyses it is expected that they will be able to exclude a low mass SM Higgs in the range $110<M_{H}<125 \mathrm{GeV}$ by the end of data taking. There is also the possibility of an observation at the $3 \sigma$ level.

\section{References}

[1] R. Barate et. al. [LEP Working Group for Higgs boson searches], Phys. Lett. B 565 (2003) 61.

[2] The LEP Electroweak Working Group, http: / / lepewwg • web. cern . ch/LEPEWWG/.

[3] Marc Buehler, "Search for a High Mass Standard Model Higgs Boson at the Tevatron", in these proceedings.

[4] See http://www-cdf.fnal.gov/physics/new/hdg/hdg.html, and http://wWW-d0.fnal.gov/Run2Physics/WWW/results/higgs.htm.

\footnotetext{
${ }^{2}$ In the two months since this conference, the main low mass analyses from CDF have all improved their sensitivities by at least $10 \%$.
} 